\title{
Mujeres en prisión. Intervención basada en sus características, necesidades y demandas
}

\author{
Concepción Yagüe Olmos \\ Directora del Centro Penitenciario de Mujeres \\ de Alcalá de Guadaíra (Sevilla)
}

\section{RESUMEN}

En las prisiones de nuestro país gestionadas por y para hombres, la mujer encarcelada ha ocupado siempre una posición muy secundaria debido a su menor entidad numérica y su falta de conflictividad. Esto ha derivado en la perpetuación histórica de una serie de factores de discriminación: precariedad de espacios, peores condiciones de alojamiento, lejanía de su entorno familiar y protector, mezcla de perfiles criminales, etc.. Y sobre todo el desconocimiento de las diferentes características, sociales y personales, que las hacen vulnerables a la entrada en el mundo penal y el desentendimiento sus necesidades. A falta de una política global de género en el ámbito penitenciario, en el Centro Penitenciario de Alcalá de Guadaíra, (Sevilla) un hemos diseñado un ambicioso programa de intervención basado en sus características, necesidades y demandas. Se propone modelo de intervención como un proceso a largo plazo de logros consolidables, desde un cierto grado de dependencia a la plena autonomía personal. (Autosuficiencia). Una combinación de programas de formación, de intervención personal y de servicios sociales que permitan a estas mujeres una mejora en sus capacidades personales, y les ayuden a ejercer plenamente sus derechos de ciudadanía. La perspectiva de género, está siempre presente, como marco de actuación.

Palabras clave: Prisión, Mujer, factores de discriminación, Programa de Intervención penitenciaria, Necesidades y demandas 


\begin{abstract}
In our country's prisons, managed by and for men, women prisoners have always occupied a secondary position due to their lower numbers and their less violent and troublesome nature. This has perpetuated throughout history a series of discriminatory factors: lack and instability of spaces for them within prisons, worse living conditions, separation and geographical isolation from their family and support networks, mixing of women with varying criminal histories, etc. Most of all, this leads to an ignorance of the different social and personal characteristics that make them vulnerable upon entering a punitive environment and creates a lack of understanding of their needs. Given that there is no overarching gender policy within the Spanish penitentiary system, nor at the Penitentiary of Alcalá de Guadaira, (Sevilla), we have designed an ambitious intervention program based on women prisoners' characteristics, needs and demands. We propose our intervention model as a long term process of consolidated achievements, proceeding from a certain level of dependency to full personal autonomy. A combination of training programs, personal and social service intervention programs that enable women to improve their personal skills, and help them fully exercise their citizenship rights. A gender perspective is always present, as a framework for action.
\end{abstract}

Keywords: prison, women, discriminatory factors, intervention program, needs and demands

\title{
Introducción
}

En nuestro país, sobre una población encarcelada que asciende a 65.539 personas el porcentaje de hombres $(91,82 \%)$ supera con creces a las mujeres $(8,18)^{\mathrm{i}}$. Desde una perspectiva objetiva recientemente están publicándose estudios y estadísticas comparativas referentes a la ejecución penitenciaria en los hombres y las mujeres poniendo de manifiesto otras muchas diferencias, más allá de su escasa representación numérica. Por ejemplo, la menor peligrosidad social femenina. Si atendemos a la actividad delictiva es el delito contra la salud pública el más frecuentemente cometido

Revista Española de Investigación Criminológica

Artículo 4, Número 5 (2007) $\quad$ www.criminología.net

ISSN: $1696-9219$ 
$(46,6 \%)$ frente a los delitos contra el patrimonio y el orden socioeconómico $(35,6 \%)$, siguiendo, muy de lejos, los homicidios y sus formas $(4,7 \%)$ y las lesiones $(2,4 \%)$, figuras, estas últimas, que implican mayor agresividad en su ejecución ${ }^{\mathrm{ii}}$. Es más importante el porcentaje de mujeres que disfrutan de un régimen penitenciario de semilibertad $(22,7 \%)$ frente al $14,4 \%$ de los hombres ${ }^{\text {iii }}$. Y si comparamos el índice de incidentes regimentales graves anuales por cada 100 internos (agresiones a otros internos, a funcionarios, autolesiones o evasiones) los hombres duplicarían a las mujeres $^{\text {iv }}$ (un $7,4 \%$ frente al $2,8 \%$ ).

No hace falta más que dar un sucinto repaso a la historia para ver que ambas características han facilitado un trato desigual a las mujeres dentro de las prisiones, con normas jurídicas que ampararon flagrantes discriminaciones (como su encarcelamiento por conductas consideradas amorales o que rompían con el papel tradicionalmente asignado a su sexo ${ }^{\mathrm{v}}$ ). Y dentro de los propios muros carcelarios, debieron soportar la concentración en unos pocos establecimientos (con el consiguiente alejamiento geográfico), una normativa mucho más restrictiva, y estar constreñidas en precarios espacios sin las más mínimas condiciones de habitabilidad.

No cabe duda de que en este último cuarto de siglo se han apreciado cambios importantes en las estructuras carcelarias de nuestro país. Desde un primer momento se pensó que igualando los estándares de opciones para hombres y mujeres se eliminaría la discriminación. Así ha sido planteado en nuestro país en el diseño de las "Prisiones Tipo", modernas estructuras que están basadas en la premisa de un género neutro: los módulos de mujeres son idénticos al resto, y tanto ellas como los reclusos podrían participar de las mismas oportunidades laborales, formativas y de ocio. Este modelo, que recoge las últimas novedades en servicios y en equipamientos sociolaborales, culturales y de ocio, ha mejorado sustancialmente la calidad de vida de sus beneficiarios.

Sin embargo, al cabo del tiempo se ha verificado la continuidad de una discriminación formal, puesto que al existir regularmente en ellos un único módulo específico para mujeres (70 aproximadamente), frente a los 12 o 14 módulos restantes para albergar a los hombres (que suelen superar los 1500), los modelos de actuación

Revista Española de Investigación Criminológica

Artículo 4, Número 5 (2007) $\quad$ www.criminología.net

ISSN: $1696-9219$ 
siguen estando diseñados para un perfil masculino. Al no haber un planteamiento en profundidad de estas políticas, en algunos aspectos la situación ha empeorado significativamente en relación a los servicios que pueden recibir, recibiendo una atención residual por su menor presencia y conflictividad. Los mecanismos de control y seguridad con que se les ha dotado (adecuados, si acaso, a un determinado perfil criminal masculino), no lo son, en absoluto, para la generalidad de las mujeres presas, y mucho menos para los hijos que comparten, en algunos casos, el internamiento con sus madres. Aún en estos centros se está muy lejos de encontrar la paridad, pues las dificultades organizativas impiden que puedan disfrutar de determinadas zonas y servicios. Así en muchos de ellos no existen espacios en las enfermerías para las mujeres; no se les permite el acceso a módulos terapéuticos; nunca se les permitirá acceder a los habituales talleres de carpintería, albañilería, etc. ${ }^{\text {vi }}$

Pero lo más preocupante es que esta falta de atención (o invisibilidad) lleva al desconocimiento de su perfil criminológico y social específico. Unas características que se están poniendo al descubierto gracias a los estudios e investigaciones impulsados por la corriente que se ha dado en llamar criminología de género, que aglutina a un número cada día mayor de hombres y mujeres que desde distintos ámbitos, académicos y en su vertiente práctica, coinciden en el empeño de destacar los factores de vulnerabilidad que empujan a las mujeres al delito, y los elementos que mantienen un trato discriminatorio dentro de las prisiones.

Es necesario acometer un profundo debate social sobre el trato que reciben las mujeres que sufren castigo penal por la trasgresión de las normas sociales, pero que a su vez son víctimas de una situación de desventaja social que se ceba en los más desfavorecidos. Por ello, voy a reflejar esta realidad haciéndome eco de los trabajos más recientes que se están llevando a cabo en nuestro país ${ }^{\mathrm{vii}}$. Desde este prisma descubrimos que su historia está repleta de factores de discriminación social anteriores a su encarcelamiento.

En el ámbito familiar, frecuentemente recae en ella la responsabilidad de los hijos no emancipados o personas dependientes, el mantenimiento de la unidad familiar o su sostenimiento económico. En el informe del Defensor del Pueblo Andaluz de $2006^{\text {viii }}$ 
encontramos que la tasa de fecundidad de las mujeres encarceladas $(2,7 \%)$ está por encima del conjunto de la sociedad española (1,3\%). El 38,7\% tienen consideración de familia numerosa, al contar con más de tres hijos. El 70\% de los hijos de estas mujeres cuentan con menos de 18 años, es decir, son menores de edad por lo que se encuentran en el periodo más vulnerable de su existencia. Con la mujer ingresada en prisión, a estos hijos no emancipados hay que buscarles acomodo, permaneciendo a cargo: del marido o compañero el 19,6\%, de la familia extensa el 38\% (los abuelos maternos fundamentalmente) o en acogimiento institucional, centro de menores o situación de preadopción o adopción el 9,5\%. Muchas de ellas son cabeza de familia monoparental (el $34,4 \%$ de estas mujeres están separadas, son solteras con hijos o viudas, frente al 19,7\% de casadas y el $36 \%$ en situación de pareja de hecho). Antes del ingreso en prisión la unidad familiar clásica, conformada por la mujer junto a su pareja e hijos se da tan solo en el 27,8\% de los casos. En prisión se encuentra el 33,3\% de sus maridos o parejas actuales. Cuando llegue el momento de su excarcelación, solo disponen de vivienda propia, arrendada o de protección oficial el 39,3\% de estas mujeres. Todos estos factores, que hubiera de tener una fuerte incidencia en la comisión del delito, se agravan irremediablemente con el encarcelamiento.

Son mujeres supervivientes a una infancia difícil, marginal, carente de oportunidades y afecto. En un $25 \%$ declaran que fueron recluidas durante determinados periodos de su adolescencia en algún tipo de institución cerrada, internado o reformatorio; generalmente por pequeños robos o consumo de drogas ${ }^{\mathrm{ix}}$. El $70 \%$ de las mujeres ingresadas admite tener algún familiar en prisión: en el 10\% se trata de algún progenitor y en el 3,3\% de alguno de sus hijos ${ }^{\mathrm{x}}$. Con una media de edad comprendida en el tramo de los 31 y 40 años, el 33,4\% ha tenido tres o más ingresos previos en prisión, siendo el índice de reincidencia el $54 \%$ en mujeres frente al $60 \%$ en hombres ${ }^{\mathrm{xi}}$

Padecen una alta tasa de analfabetismo (a nivel nacional, el $48,7 \%$ se declara analfabeta o sin estudios primarios a su ingreso en prisión ${ }^{x i i}$; en Cataluña aproximadamente el $50 \%$ de las mujeres no tienen el certificado de escolaridad o asistieron a la escuela menos de 8-9 años y tan solo el $11 \%$ cursaba algún tipo de estudio superior ${ }^{\text {xiii }}$ ). En el área económica destaca una ínfima cualificación profesional o experiencia laboral, pues el 11,5\% nunca desempeñó un trabajo remunerado, y las que sí

Revista Española de Investigación Criminológica

Artículo 4, Número 5 (2007) $\quad$ www.criminología.net

ISSN: $1696-9219$ 
lo hicieron lo desarrollaron mayormente en labores de limpieza, venta ambulante, y como temporeras en el campo ${ }^{\text {xiv }}$. Es decir, reúnen todas aquellas características que las hacen menos competentes socialmente, las predisponen al desempleo o las ocupaciones peor remuneradas.

$\mathrm{Su}$ estabilidad mental está comprometida por la incidencia de problemas psíquicos (26\%), sobre medicación (16,5\%) y el abuso de sustancias adictivas (alcohol en el $8,1 \%$, y otras drogas de abuso en el $42,1 \%)^{\mathrm{xv}}$. En el aspecto sanitario la prevalencia de VIH es del $22,1 \%$ (frente al 17,3 de los presos varones) y del $28 \%$ de Hepatitis C (frente al $32 \%$ en hombres) ${ }^{\text {xvi }}$.

Muchas de ellas pertenecen a minorías desfavorecidas, como se confirma con la sobrerepresentación numérica de mujeres de etnia gitana y extranjeras $(16,1 \%$ y $35 \%$ respectivamente $\left.{ }^{\mathrm{xvii}}\right)$.

En el trato diario, directo y personal, confirmamos esta falta de competencia social, traducido en la ausencia de habilidades sociales, en su aspecto personal descuidado, carencia de pautas cívicas básicas: ademanes, caudal de voz, educación, ahorro, hábitos alimenticios, etc.

Mujeres cuya autoestima es muy baja, pues perciben el fracaso de sus propias expectativas $^{\text {xviii }}$, acerca de su papel como mujeres, como madres, esposas. Su autoconcepto es muy negativo pues, además, el estigma de la prisión las lleva a verse como menos capaces y valiosas.

La temprana exposición a modelos delincuenciales provoca una adquisición de valores asociales donde la fuente de ingresos no pasa por el sacrificio, ni el esfuerzo personal. La subsistencia se encomienda al azar o a los servicios sociales. Las gratificaciones han de ser inmediatas. La ambición, y la envidia hacia el éxito fácil están muy presentes.

Mujeres que sufren una dependencia psicológica de la figura masculina, y una falta de autonomía personal. Siempre supeditada a la pareja actual, sin cuyo concurso se siente incompleta o fracasada.

Hoy sabemos de la altísima frecuencia de episodios de abusos sexuales y maltrato familiar y de pareja en el historial de las mujeres encarceladas. En la más completa y reciente investigación del grupo SURT ${ }^{\mathrm{xix}}$ se reseña que el porcentaje de mujeres que ha sufrido algún tipo de violencia asciende a la escalofriante cifra de 88,4\%. Según 
tipología, la violencia de tipo sexual afectaba al 68\% (dentro de ésta, el $41 \%$ se había producido de forma sistemática, el $59 \%$ en el ámbito familiar y el 25\% sufrieron estos abusos siendo menores). La violencia física afectaba al $74 \%$ de los casos (de estos, el $80,4 \%$ de las mujeres encarceladas habría sufrido malos tratos o situaciones objetivas de violencia en el ámbito doméstico, cuando en el conjunto de la sociedad esta cifra se sitúa alrededor del $\left.12,4 \%{ }^{\mathrm{xx}}\right)$. También conocemos la relación directa que estas experiencias han tenido en su historial delictivo y las consecuentes secuelas físicas y psicológicas que acarrean, como el trastorno de estrés postraumático (PTSD), una enfermedad frecuente derivada de experiencias de violencia en la infancia y juventud, que se caracteriza por problemas de sueño, irritabilidad, drogodependencia, depresión e intentos de autolisis, agresividad, sobremedicación, etc. ${ }^{x i}$

Completando este panorama, hay una circunstancia fundamental que tiñe especialmente el ingreso de cualquier mujer en la prisión, pues si para un hombre, su encarcelamiento tiene consecuencias graves en el ámbito familiar, cuando se trata de una mujer, madre de familia, este hecho es vivido por ellas como un auténtico cataclismo. La mujer, a su ingreso, en su bagaje personal trae consigo la culpabilidad, la angustia e incertidumbre por la responsabilidad familiar en el exterior: hijos, padres o personas dependientes e incapacitadas; la pérdida de la vivienda, o la inseguridad hacia el mantenimiento de la estabilidad marital, etc. La impotencia ante la previsión de consecuencias irreversibles en la red familiar por su encarcelamiento; sobre todo les pesa su ausencia en las enfermedades, o ante la adolescencia de los hijos, que pueden verse encaminados a la drogodependencia, o a engrosar pandillas o redes delictivas.

Vemos, en fin, que las mujeres son diferentes, y viven su encarcelamiento de forma diferente y por ello hay que promover para ella medidas específicas. Ordinariamente se olvida el mayor sufrimiento que provoca su ingreso en prisión al entender que están desatendiendo sus obligaciones familiares.

No es nueva la idea de que para atajar las discriminaciones históricas sufridas por ciertos colectivos se hace necesario implantar medidas de acción positiva, encaminados a igualar las diferencias. Hasta este momento, no se ha impulsado una estrategia general penitenciaria de género ni se han implementado programas 
específicos generales dirigidos a ellas. El desconocimiento al que aludimos ha hecho que, de forma generalizada, se hayan desatendido sus particulares opiniones y anhelos. Faltan programas concretos, basados en sus diferencias de género, que contemplen cuales son los caminos y las vías de marginalidad por las que algunas de estas mujeres, acaban ingresando en el sistema carcelario.

\section{Filosofía de una intervención penitenciaria}

Es por todo ello que en el Centro Penitenciario de Alcalá de Guadaíra $(\text { Sevilla })^{\text {xxii }}$ hemos diseñado una intervención penitenciaria basada en el profundo conocimiento de las características, necesidades y demandas de estas mujeres, que hemos ido adquiriendo a lo largo de más de quince años de trabajo en estrecha convivencia con ellas.

Desde un primer momento nos situamos en el debate de definir la finalidad de nuestro trabajo, esto es, cuál debe ser el objeto de nuestra intervención en prisión. Si atendemos al requerimiento formal de nuestro ordenamiento, además de las funciones de custodia, nuestra Constitución nos ordena orientar nuestro esfuerzo hacia la reeducación y reinserción social. En el sentir de la ciudadanía parece que el éxito de nuestra labor estará ligado a un factor primordial: reducir la probabilidad de reincidencia. Y simplificando, hablaríamos de lograr la plena reinserción social cuando fuéramos capaces de garantizar a cada interna que abandonase la prisión, un trabajo digno y suficientemente remunerado con el que pudiera satisfacer convenientemente sus necesidades personales y familiares. En la posición utópica de que esto fuera factible, cuantas de aquellas mujeres serían capaces, al cabo de unos pocos meses, de ....mantenerlo.... Ya hace tiempo que los responsables de programas sociales tomaron conciencia de que, a pesar de contar con posibilidades reales de empleo, algunas de las características personales de los sujetos interferían o suponían obstáculos insalvables a la hora de culminar con éxito la vía de la inserción laboral.

Cualquiera de nosotros ha pasado por un proceso de socialización en la escuela, dónde adquirió unos ciertos hábitos de responsabilidad y sacrificio: puntualidad, orden, 
disciplina, hábitos de respeto a los demás, limpieza, habilidades en el trato, cuidado del aspecto personal, etc. En la adolescencia, recibimos una atención especial en la asunción de valores prosociales y la evitación de conductas perniciosas y adicciones. Tras todo esto, un aprendizaje en destrezas profesionales, una especialización laboral. En los primeros trabajos temporales aprendimos a desenvolvernos profesionalmente de forma gradual: a recibir órdenes, a trabajar en equipo, a sufrir contrariedades, etc., contando, casi siempre, con el apoyo y consejo de la familia cuando las cosas no salen bien, y la alegría compartida en los logros.

En la mayoría de las mujeres que tratamos a diario, este proceso quedó interrumpido en alguno de estos pasos: abandono escolar, desapego o ruptura familiar, etc.. Y en el camino fueron quedando atrás las posibilidades ordinarias de desarrollo, y lo que es muy común, la pérdida del control sobre su propia vida, cayendo en todo tipo de dependencias: de la droga, del delito, de la obtención de dinero sin esfuerzo, de parejas tóxicas. De ahí surge la disyuntiva REEDUCACIÓN vs EDUCACIÓN, es decir, asumir que, en muchos casos, nuestra tarea supondrá retomar el camino desde las primeras fases del desarrollo.

\section{Metodología de la intervención penitenciaria}

Definiríamos nuestro modelo de intervención como un proceso a largo plazo de logros consolidables, desde un cierto grado de dependencia a la plena autonomía personal (autosuficiencia). Se trata de una combinación de programas de formación, de intervención personal y de servicios sociales que permitan a estas mujeres una mejora en sus capacidades personales, y les ayuden a ejercer plenamente sus derechos de ciudadanía. La perspectiva de género, está siempre presente, como marco de actuación.

Tomamos una escalera ${ }^{\text {xxiii }}$ como modelo. Tratamos de situar a cada interna en el lugar de la escalera que le corresponde, tutelarla, y acompañarla hacia el alcance de sus logros particulares. Tenemos que poder ofrecer a cada persona lo que necesita, sea lo que sea. Para eso hay que empezar por el sitio en que está situada cada una de ellas, y valorar y recompensar cada paso adelante. 
El objetivo es estar en la escalera, y tener un plan para subir los peldaños. Intentar crear y facilitar la misma progresión, y el entorno social que ha experimentado una persona que ha logrado la autonomía y el éxito social, por ejemplo, un trabajo digno, una estabilidad familiar, etc. Los primeros escalones son los más costosos y trascendentes. Por eso es sumamente importante conseguir implicar a cada interna en la mejora de sus competencias, donde asuma el protagonismo a la hora de fijar sus metas a corto, medio y largo plazo.

Método: el acompañamiento. Debemos convertirnos en agentes activos de socialización. La simple oferta de actividades, programas y servicios no vale por sí sola, puesto que oportunidades similares se le ofrecieron en el exterior y no fueron aprovechadas. Nuestra presencia ha de ser continua: en la elaboración conjunta de su plan inicial de acción y en sus futuras revisiones, y en el seguimiento de su quehacer diario, tutelando sus avances, corrigiendo errores, remarcando los aciertos, motivando el cambio...

Es imprescindible haber creado en la prisión un ambiente seguro y ordenado, un sistema organizativo con normas de convivencia claras, previsibles, que minimice la tensión y reduzca las posibilidades de incidentes. Un sistema que enfatice las relaciones interpersonales positivas entre las compañeras y entre las internas y los miembros de personal, basadas en el respeto mutuo. Un entorno que potencie las muestras de afecto, el sentido del humor, y la cercanía hasta perderle el miedo a la calidez del contacto físico.

La evaluación de este programa, lo será en clave de las mejoras incrementales que se vayan obteniendo. Un avance cuantificable en el grado de consecución de los objetivos planificados. Tenemos que estar en disposición de medir los avances que se vayan consolidando en las diversas áreas de actuación: social, personal, educativa/ laboral y en los programas de inserción, que se definirán a continuación. 


\section{La intervención centrada en sus necesidades}

A) En primer lugar, antes de iniciar cualquier otra intervención, la atención prioritaria del equipo de tratamiento está enfocada en resolver las problemáticas sociofamiliares que desencadenan el ingreso en prisión. Es la única forma de paliar la situación de angustia y estrés que tiñe toda su existencia, e interfiere en su capacidad de implicación en las demás tareas.

a) Orientación legal e información actualizada de su situación procesal, penal y penitenciaria, que les permita trazar unas expectativas realistas de futuro a medio y largo plazo.

b) Actualización de la Documentación, personal y familiar, necesaria para ejercer plenos derechos de ciudadanía. Es sumamente frecuente el deterioro, extravío o inexistencia de registros de nacimiento, DNI, cartilla sanitaria, libro de familia, etc., lo que, en la práctica, les impide participar de los recursos sociales como ciudadanas de pleno derecho.

c) Acceso a ayudas y asesoramiento de los cauces y servicios sociales existentes. Acceso subsidios, a ayudas para la vivienda, escolarización, becas, subvenciones, etc..

d) Procedimientos de familia: ayuda y asesoramiento legal en los procesos de acogimiento, adopción u oposición a medidas de protección de sus hijos.

e) Iniciar, con la mediación de los servicios sociales y el personal de tratamiento, la restauración de los vínculos familiares deteriorados o rotos para recomponer el tejido social de procedencia. En su defecto, la búsqueda de recursos sociales alternativos de asistencia con vistas a su paulatina reintegración social.

f) Potenciar redes de apoyo entre las compañeras de internamiento. Crear un sistema de acogida y tutela con internas especialmente preparadas con el objeto de crear a su alrededor un entorno protector que les sirva de guía en el conocimiento de la normativa e influir positivamente en su integración en la vida del centro. 
B) En segundo lugar, y como hemos mencionado, muchas de estas mujeres atravesaron la infancia y la juventud sin adquirir unas destrezas básicas para organizar su vida ordenadamente. Hemos de trabajar, por tanto estas capacidades.

a) Hábitos de higiene (personal y de su espacio), de salud (luchar contra los malos hábitos, supersticiones), de alimentación. Revisión diaria de las habitaciones, de su aseo personal, control de la dieta, control sanitario, etc.

b) Normas fundamentales de convivencia: respeto a la propiedad privada, uso responsable de las instalaciones y materiales comunitarios, orden en las actividades comunes (por ejemplo, respetar las colas del economato y comedor), no tirar los vasos y papeles al suelo, etc. Para ello, en los talleres formativos se trabaja en la confección de su propio hábitat. Casi todas ellas participan en la confección de la lencería y ornamentos de decoración de dormitorios y espacios comunes, en la pintura de habitaciones o sus muebles, etc.; todo ello supone un elemento motivador muy potente, ya que el orgullo hacia los propios logros facilita el estado de conservación de los mismos.

c) Habilidades básicas para el mantenimiento de una actividad regular: puntualidad, compromiso, rendimiento, resistencia a la frustración, paciencia; para ello son útiles los talleres ocupacionales, donde iniciarse en tareas sencillas y rutinarias.

d) Adquisición de destrezas motrices esenciales para el desarrollo de cualquier actividad: prensión, precisión, (previas a poder coger un lápiz, al manejo de una herramienta, etc. En mujeres muy deterioradas es frecuente recurrir a las "tareas de aguja": punto de cruz, bordado, bolillos, etc. que ayudan a adquirir o mejorar las capacidades motoras). ${ }^{\text {xxiv }}$

C) En el campo de la Educación cubrimos casi todos los niveles educativos fundamentalmente los niveles de alfabetización y educación base. El grado de aceptación y motivación es altísimo, pues algunas de ellas descubren en la escritura una forma nueva de comunicarse con los suyos y con el mundo que les estaba vedada. ${ }^{x x v}$ 
La formación profesional se imparte mediante cursos de informática, confección, decoración de azulejos y peluquería. En las tareas formativas, el enfoque suele volcarse de nuevo hacia las actividades femeninas: labores, peluquería y estética. Es la principal crítica que se recibe desde la observación externa. También es cierto que son actividades que pueden promover el autoempleo, y por este motivo las más demandadas por ellas que conocen bien sus propias limitaciones y el papel social al que han de retornar.

La contratación de monitores permite garantizar la promoción cultural, deportiva y artística: teatro, cerámica, animación a la lectura, actuaciones musicales, etc.

Como consecuencia de su perfil social, buscamos atender a otros colectivos que dentro de esta población pueden padecer un mayor índice de marginalidad. Nos referimos a la sobre representación de internas de etnia gitana y extranjeras, con programas de integración cultural, aprendizaje de español, etc.

D) Como actividad laboral, 24 internas disfrutan de trabajo remunerado a través de empresas externas: confección y manipulados de cerrajería, y otras 12 internas disponen de trabajo a cargo de la Institución Penitenciaria: cocina, lavandería, economato, etc. Para internas que están próximas a su puesta en libertad existen recursos (hoy aún muy escasos) de inserción laboral, con becas de formación remunerada, y contratos en hostelería: lavanderías, restaurantes, etc. o en el recurrido servicio doméstico.

E) Otra de nuestras principales preocupaciones es la mejora de su independencia-autonomía personal. En este campo de intervención se incluyen programas específicos enfocados a disminuir la vulnerabilidad de la mujer reclusa, en general, a sufrir situaciones de violencia y/o dependencia.

\section{E)-1 Prevención del maltrato.}

La forzosa separación de la figura dominante masculina nos permite abrir un nuevo espacio de reflexión. La observación de otros modelos femeninos, autosuficientes 
o independientes (otras compañeras, profesionales y las propias funcionarias) sirve de punto de apoyo para su redescubrimiento. Con asesoramiento de asociaciones externas, se han implantado programas de enorme aceptación para mujeres que son, o fueron, víctimas de violencia de género (programa que denominamos "porque yo lo valgo" para disminuir su carga negativa). Se basa en actividades de desarrollo cognitivo sobre los factores culturales que sustentan la desigualdad sexual: análisis de mitos y prejuicios ligados al sexo y al género; de prevención de la violencia y/o explotación sexual; desarrollo de la independencia económica; de mejora de la autoestima, etc.

\section{E)-2 Promoción de hábitos saludable y atención a las drogodependencias}

La alta incidencia de la dependencia de sustancias adictivas, es un factor frecuente de la población reclusa, y estos programas están regularmente implantados en la totalidad de las prisiones, contando con los recursos comunitarios específicos. En lo que respecta a las mujeres, la drogodependencia cursa, además, con una alta sintomatología depresiva, con alto riesgo de autolisis, padecimientos físicos y psiquiátricos, e irritabilidad, sintomatologías que están frecuentemente en correlación con su historial pasado de violencia familiar, y abusos sexuales (estrés postraumático). Son mujeres vulnerables con una gran necesidad de tratamiento. Son condicionantes anteriores a su entrada en prisión, y que se agravan en ella.

Esta atención se completa con actividades regulares de promoción de la salud, entre los que se incluye un novedoso programa de deshabituación tabáquica en concurso con responsables sanitarios de distrito y del hospital de referencia (Valme). Es una terapia multicomponente (motivación, sesiones de grupo, prescripción parches transdérmicos de nicotina, control coximétrico, etc.) a la que asisten en igualdad de condiciones las internas del centro y los trabajadores, motivo éste de una extraordinaria fuerza de feed-back positiva entre ambos grupos.

\section{E)- 3 Programa de jóvenes}

Atendiendo a las características peculiares de esta población ${ }^{\mathrm{xxvi}}$, la entrada en prisión de las mujeres jóvenes suele constituir el primer parón importante en la espiral de consumo, actividad delictiva y marginalidad de sus precoces vidas. La intervención se basa en reanudar el interrumpido proceso madurativo y de socialización; en suplir la 
ausencia generalizada de referentes morales que por distintos motivos no estuvieron presentes en su desarrollo, y en paliar las fuertes carencias educativas y formativas.

Partimos de la voluntariedad y expresa aceptación de la joven involucrada, pues todo este programa va a suponer un altísimo nivel de exigencia y compromiso por su parte y de las demás personas implicadas. El refuerzo se sitúa en la superación de unas etapas que culminarán con su integración prioritaria en la vida educativa y laboral del centro y su rápida inclusión en programas de inserción en comunidad. Los pilares de esta intervención se encuentran en dos figuras que adquieren un fuerte protagonismo. La primera es la asignación de una interna de apoyo que ejercerá de figura "maternal"xxvii bajo la supervisión y apoyo constante de los miembros del equipo de tratamiento. La segunda es un tutor profesional, que, mediante técnicas similares a las terapias sistémicas, llevará a cabo reuniones individuales con la menor, con ésta y la tutora, y reuniones grupales, corrigiendo las desviaciones y reforzando los avances.

E)- 4 Mujeres mayores, discapacitadas físicas o psíquicas. Sin ser un lugar adecuado, constatamos que muchas veces ante la falta de respuesta de otras instituciones o servicios sociales, la prisión se ha convertido en un recurso social muy valioso para atender a estos perfiles. Nuestro esfuerzo se encamina a mejorar su calidad de vida y a trabajar en la mejora de su autonomía física y personal. Mediante internas tutoras especialmente entrenadas se impulsa, para cada una de estas mujeres, un programa paulatino de aprendizaje en habilidades motoras y de auto cuidados en higiene y salud, pretendiendo conseguir la máxima autonomía e independencia en las tareas ordinarias de su vida cotidiana.

\section{E)- 5 Desarrollo de la maternidad}

Finalmente, hemos de contemplar la principal particularidad de los establecimientos femeninos: el desarrollo de la Maternidad dentro de la prisión. La "Escuela de madres" cubre un ambicioso programa que va desde actividades complementarias de educación base, a formación psicopedagógica o talleres ocupacionales, a cargo de personal especializado y mediante convenios con alguna ONG. En esta escuela suelen participar voluntariamente los más prestigiosos 
profesionales de la provincia: ginecólogos, pediatras, matronas, psicólogos, etc., en un acercamiento al conocimiento de la maternidad en todas sus facetas. Otras ONG colaboran en actividades complementarias de ocio para los niños y programan frecuentes salidas a campamentos y/o excursiones.

\section{Evaluación de los programas}

Dada la complejidad y diversidad de la actuación en este centro con un alto número de programas implantados, uno de los principales retos que se nos plantea es la validación de los resultados obtenidos para poder valorar la incidencia real en la mejora de las condiciones de estas mujeres, a medio y largo plazo. Esto es, no solo determinar el éxito de cada intervención, sino su incidencia real en la bajada de los índices de reincidencia de las mujeres sometidas a estas intervenciones y la resolución de la problemática anterior a su ingreso.

Los equipos de tratamiento que trabajan en las prisiones destacan por su voluntariedad, entrega y espíritu innovador a la hora de importar experiencias y buenas prácticas al ámbito penitenciario. Sin embargo, una dificultad común que nos aqueja a los profesionales de este campo es la falta de formación en instrumentos de control y validación. Se trabaja mucho, pero sin contar con instrumentos contrastados lo que lastra definitivamente la fiabilidad de los resultados obtenidos, por más que la impresión general de este gran esfuerzo sea muy positiva.

Es relativamente fácil seguir el efecto parcial de estas intervenciones si medimos las mejoras incrementales en la consecución de los objetivos particulares que se han ido proponiendo $^{\text {xxviii }}$ y por ello citamos algunos resultados a modo de ejemplo. En el área escolar, la memoria de la Unidad Educativa del curso 2005/2006 señala un índice de consolidación formal de conocimientos en superior al $90 \%$ en enseñanzas iniciales (alfabetización y neolectores y educación base). En el ámbito de la intervención psicosocial, el programa de jóvenes es el que obtiene mejores resultados, pues desde el momento de su implantación, en Febrero de 2006, hasta la fecha de control, en Junio 2007, el 31,3\% de ellas completaron las etapas que se les habían fijado culminando con su inserción laboral en talleres productivos del centro ${ }^{\text {xxix }}$ (lo que en muchos casos 
suponía la primera experiencia laboral contractual de su vida). En el área sanitaria el programa de deshabituación tabáquica, presenta unos índices de éxito (persistencia del no consumo al cabo de un año desde el inicio de la terapia) del 22,7\% en el grupo de internas y del $100 \%$ en el de trabajadores.

Los resultados globales obtenidos fluctúan entre dos extremos opuestos. Desde aquellos casos de internas sumamente deterioradas en los que el periodo de prisión impuesto no nos permite alcanzar más que una mejora en sus condiciones sanitarias, en aspecto personal (consecuencia de una vida controlada y ordenada en horarios, alimentación y atención médica), y una breve consolidación de conocimientos en la esfera escolar y formativa, a aquellos otros en que pueden apreciarse transformaciones realmente espectaculares. Mujeres que descubren potenciales ocultos bajo una vida apremiante $\mathrm{y}$ desordenada que nunca hubieran sospechado ${ }^{\mathrm{xxx}}$. O muchachas que posiblemente hubieran estado abocadas a una espiral de drogas, agresiones y pérdida de lazos familiares, que reconducen su vida positivamente ${ }^{\mathrm{xxxi}}$. Con toda esta suma de iniciativas confiamos en ayudar a reconstruir su situación en sociedad como mujeres y ciudadanas de pleno derecho. Pero además, en el papel que ejercen como madres la actuación sobre estas mujeres ha de tener un efecto positivo a largo plazo: la "sinergia positiva" hacia su ámbito de referencia, sobre todo en relación a sus hijos, para los que son capaces de abrir unas nuevas perspectivas.

En palabras de Frieder Dünkel ${ }^{\mathrm{xxxii}}$ "los meta-análisis de la investigación sobre el tratamiento de las mujeres confirma que un régimen orientado a las necesidades de las internas conducen a importantes resultados, mostrando una efectividad de entre $.22 \mathrm{y}$ .38 en los siguientes ámbitos: en los comportamientos antisociales y los déficits en la capacidad de actuar(.31), en la falta de autocontrol (.22), en los problemas en el ámbito intrafamiliar u otros problemas en la resolución de conflictos interpersonales (.30), etc.. Los mejores resultados los producen los programas que se centran en los problemas interpersonales y especialmente en las variables familiares. Además, se demuestran especialmente significativos para la rehabilitación los programas de tratamiento que se centran en la victimización anterior, la traumatización y en el aumento de la autoestima." 
Es el objeto de nuestro trabajo una intervención volcada en sus necesidades contrastadas, lo que auguraría en esta línea unos resultados, al menos, similares a los indicados, máxime si tenemos en cuenta que el nivel de participación de la totalidad de las internas del centro en algunas de estas actividades se sitúa alrededor del 98\%, y que más del $85 \%$ interviene en tres o más de estos programas simultáneamente. Es previsible que, a corto plazo, implementemos sistemas que nos permitan medir correctamente los índices de éxito, pues con este objeto se están concertando convenios de colaboración con universidades que cuentan con departamentos especializados en la evaluación de programas sociales, para medir la eficacia real de los mismos. 


\section{Referencias}

1. ALMEDA SAMARANCH, ELISABETH. Mujeres Encarceladas. Ed. Ariel. 2003

2. ALMEDA SAMARANCH, ELISABETH Corregir y castigar, el ayer y hoy de las cárceles de mujeres. Ed. Bellaterra. 2002

3. ALMEDA SAMARANCH, ELISABETH. "Las experiencias familiares de las mujeres encarceladas". Familias de ayer, familias de hoy : continuidades y cambios en Cataluña. Coordinado por Xavier Roigé Ventura. Icaria 2006

4. AZAOLA E. "Prisiones para mujeres: un enfoque de género." Programa interdisciplinario de Estudios de la Mujer. México 1994.

5. BARBERET, R. Y MIRANDA, M.J. Análisis de la eficacia y adecuación de la política penitenciaria a las necesidades y demandas de las mujeres presas. (PTE. de publicación).

6. BERTRAND M.A. Prisons pour femmes. Montréal: Les Éditions du Méridien, 1998, 449 pp.

7. CENTRE D'ESTUDIS JURIDICS "La opinió de les dones recluses a Catalunya sobre l'encarcelament”. Estadística básica de la justicia a Catalunya nº 45. Nov. 2006

8. COYLE, A. "La admunistración Penitenciaria en el contexto de los derechos humanos. Manual para el personal penitenciario". International Centre for Prison Studies.2002 (Reclusas, Pp. 131136)

9. DEFENSOR DEL PUEBLO ANDALUZ. Mujeres privadas de libertad en centros penitenciarios de Andalucía. En red, http://www.defensor-and.es/informes/ftp/mujeres_presas.pdf.

10. DÜNKEL F., KESTERMANN C., ZOLONDEK J., "Estudio internacional sobre el régimen penitenciario de mujeres. Evaluación, estudio de necesidades y mejores prácticas". Universidad Greifswald. 2005

11. EQUIPO MIP (MUJERES INTEGRACIÓN Y PRISIÓN) CRUELLS, M. IGAREDA, N. TORRENS, M. Y CRUELLS, E. "Un análisis de los procesos de integración sociolaboral de las mujeres presas en Europa”. Informe nacional España. 2004

12. EQUIPO MIP (MUJERES INTEGRACIÓN Y PRISIÓN) CRUELLS, M. IGAREDA, N. "Informe nacional de recomendaciones y propuestas políticas". 2005

13. GENERALITAT DE CATALUNYA. Centre d'Estudis Jurídics i Formació Especialitzada. L'opinió de les dones recluses a Catalunya sobre l'encarcerament. Justitada .Estadística básica de la justicia a Catalunya. $\mathrm{N}^{\circ} 45$ Noviembre 2006.

14. GENERALITAT DE CATALUNYA. Les dones a les presons de Catalunya. $\mathrm{N}^{\circ} 1$ Diciembre 2006. Secretaria de Serveis Penitenciaris, Rehabilitació i Justícia Juvenil. Àrea de Planificació i Projectes Estratègics. Serveis Penitenciaris i Rehabilitació.

15. GIMÉNEZ SALINAS, ESTHER "Mujeres Delincuentes: del mito a la prisión". Revista del Poder Judicial 48. 1997, pp. 267-288.

Revista Española de Investigación Criminológica

Artículo 4, Número 5 (2007) $\quad$ www.criminología.net

ISSN: $1696-9219$ 
16. HERR, TOBY y L. WAGNER, SUZANNE, Beyond Barriers toWork A Workforce Attachment Approach That Addresses Unpredictability, Halting Progress, and Human Nature. Project Match 2007. En red http://www.pmatch.org/barriers_feb_ohseven.pdf.

17. LARRAURI, ELENA "la mujer ante el derecho penal" Revista de la Asociación de ciencias penales de Costa Rica. $\mathrm{n}$ 11, 1996. En red: http://www.poderjudicial.go.cr/salatercera/revista/REVISTA\%2011/larrau11.htm

18. LAURENZO COPELLO, P. "La discriminación por razón de sexo en la legislación penal." Jueces para la Democracia. $N^{\circ} 34$. Marzo 1999, pp. 16-23.

19. MARTÍNEZ GALINDO, GEMA Galerianas, corrigendas y presas. Nacimiento y consolidación de las cárceles de mujeres en España (1608-1913). Edisofer. Madrid, 2002.

20. MIRANDA LÓPEZ, M. J. "Delincuencia femenina y situación social de las mujeres". Las mujeres y la cuidadanía en el umbral del siglo XXI. Universidad complutense1998. Pp. 249-252.

21. MIRANDA LÓPEZ, M. J. "Cárceles ¿Para qué? Política y sociedad. Vol. 39, nº 2. 2002, pp. 377-398

22. MORRIS, A., WIKILSOSON, C., y OTROS. "La atención a las necesidades de las mujeres encarceladas". Home office, UK. (traducido por Aguilar de los Santos, R.)

23. PARLAMENTO ANDALUCÍA. DIARIO SESIONES COMISIÓN DE IGUALDAD Y BIENESTAR SOCIAL. "Informe especial del Defensor del pueblo andaluz relativo a mujeres privadas de libertad en centros penitenciarios de Andalucía” y "Proposición no de Ley relativa a actuaciones dirigidas a la población de mujeres reclusas”. 19 de Diciembre 2006.

24. RIVAS N., ALMEDA E., BODELÓN E., RASTREANDO LO INVISIBLE. Mujeres extranjeras en las cárceles. Anthopos. 2006

25. SURT. CRUELLS, M., TORRENS M., IGAREDA, N. "Violencia contra las mujeres. Análisis en la población penitenciaria femenina”. 2005. En red: http://www.surt.org/cast/docs/estudio_final_cast.pdf

26. YAGÜE OLMOS, CONCEPCIÓN. "Madres en Prisión. Historia de las cárceles de mujeres a través de su vertiente maternal" Ed. Comares 2007

27. YAGÜE OLMOS, CONCEPCIÓN "Las Mujeres encarceladas". Mujeres en la periferia. Algunos debates sobre género y exclusión social" Icaria 2006

28. YAGÜE C. Y CABELLO, M. I, "las Mujeres jóvenes en prisión”, en Revista de Estudios de Juventud: Jóvenes y prisión., $\mathrm{n}^{\circ}$ 69. 2005, pp. 29-48.

29. YAGÜE OLMOS, CONCEPCIÓN. "Mujeres encarceladas" Revisión del libro del mismo nombre de Elisabeth Almeda. Revista Electrónica de investigación Criminológica (REIC)” 2003

Revista Española de Investigación Criminológica

Artículo 4, Número 5 (2007) $\quad$ www.criminología.net

ISSN: $1696-9219$ 
${ }^{\text {i }}$ Cifras de población encarcelada a 30 de Mayo de 2007. Servicio de Estadística de la Dirección General de Instituciones Penitenciarias.

http://www.mir.es/INSTPEN/INSTPENI/Gestion/Estadisticas_mensuales/2007/05/

ii En los hombres, los delitos más frecuentes son: contra el patrimonio y el orden socioeconómico $(43,48 \%)$, el delito contra la salud pública $(26,47 \%)$, contra la libertad sexual $(6,46)$, los homicidios y sus formas $(5,27)$ y las lesiones $(4,92 \%)$. Datos de elaboración propia a través de la estadística de tipología delictiva de la población reclusa penada por Ley Orgánica 10/95. Fuente, DGIIPP, ídem referencia $n^{\circ} 1$.

iii Ídem 1. Distribución de la población reclusa penada por grados de tratamiento. Estas cifras aumentan significativamente en la comunidad catalana, única con competencias administrativas asumidas, con tasas de semilibertad del 40,2\% en mujeres y $26,1 \%$ en hombres, en "Las mujeres en las prisiones de Cataluña”. Diciembre 2006, pág. 25.

${ }^{\text {iv }}$ Extrapolando los datos de conflictividad del año 2006 en la comunidad de Cataluña sobre el resto del territorio nacional. En "Les dones a les presons de Catalunya" Servicio de Estadística de la Generalitat de Catalunya. Pág. 38.

v Como ocurría, por ejemplo con la persecución de la prostitución, el adulterio, la desobediencia marital, etc., difícilmente aplicables a los hombres. Para profundizar en la evolución histórica del encarcelamiento femenino en nuestro país son imprescindibles los trabajos de Gema Martínez Galindo, Galerianas, Corrigendas y Presas; de Elisabet Almeda, Corregir y castigar, el ayer y hoy de las cárceles de Mujeres y el reciente trabajo de la autora Madres en Prisión. Historia de las cárceles de mujeres a través de su vertiente maternal.

${ }^{\text {vi }}$ Así se recoge en el acertado informe especial del Defensor del Pueblo Andaluz de 2006 "Mujeres Privadas de Libertad en los centros Penitenciarios de Andalucía". http://www.defensorand.es/informes/ftp/mujeres_presas.pdf

vii Me referiré a los siguientes trabajos: 1.- "Las experiencias familiares de las mujeres encarceladas". Familias de ayer, familias de hoy : continuidades y cambios en Cataluña, de Elisabel Almeda, a través de entrevistas en profundidad a 31 mujeres en un trabajo de campo efectuado en la prisión de mujeres de Brians en 1995. 2.- El informe Mujeres privadas de libertad en centros penitenciarios de Andalucía de la Oficina del Defensor del Pueblo Andaluz, con entrevistas a una muestra aleatoria de 61 internas ingresadas en los 10 centros de esta Comunidad que albergan mujeres. 3.- Informe del servicio de estadística de la Generalitat de Catalunya "Les dones a les presons de Catalunya" sobre la totalidad de reclusas de esa comunidad en Diciembre de 2006. 4.- Informe de la Generalitat de Catalunya "L'opinió de les dones recluses a Catalunya sobre l'encarcerament" con cuestionarios cumplimentados por 89 internas de los establecimientos de Brians y Dones de Barcelona en 2004. 5.- Del Grupo SURT, "Violencia contra las mujeres. Análisis en la población penitenciaria femenina" de 2005 sobre una muestra de 200 internas de los centros Penitenciarios de Cataluña. 6.- Dünkel, Kestermann y Zolondek "Estudio internacional sobre el régimen penitenciario de mujeres. Evaluación, estudio de necesidades y mejores prácticas" sobre una muestra de 653 mujeres distribuidas en 19 centros de 9 países (89 de

Revista Española de Investigación Criminológica

Artículo 4, Número 5 (2007) $\quad$ www.criminología.net

ISSN: $1696-9219$ 
España, las mismas encuestas cedidas para el estudio de la Generalitat arriba mencionado). 7.- Y Concepción Yagüe, "Las Mujeres encarceladas" en Mujeres en la Periferia, con datos elaborados a través de la estadística de la totalidad de la población en prisión en España en Julio de 2005.

viii Informe de la Oficina del Defensor del Pueblo Andaluz, Mujeres privadas de libertad en centros penitenciarios de Andalucía. Pág. 129-140

${ }^{\text {ix }}$ Elisabel Almeda "Las experiencias familiares de las mujeres encarceladas". Familias de ayer, familias de hoy : continuidades y cambios en Cataluña. Pág. 73

${ }^{x}$ Defensor del Pueblo Andaluz Mujeres privadas de libertad en centros penitenciarios de Andalucía. Pág. 133.

xi “Las Mujeres encarceladas” en Mujeres en la Periferia, de Concepción Yagüe. Pág. 136.

xii Datos elaborados sobre la estadística del nivel de instrucción académico del curso escolar 2004-2005. Informe General 2005. Dirección General de IIPP. Pág. 117.

xiii Dünkel, Kestermann y Zolondek "Estudio internacional sobre el régimen penitenciario de mujeres. Evaluación, estudio de necesidades y mejores prácticas”. Pág. 22

${ }^{\text {xiv }}$ Defensor del Pueblo Andaluz Mujeres privadas de libertad en centros penitenciarios de Andalucía. Pág. 141.

${ }^{x v}$ Según declaraciones al ingreso en prisión, recogidas en las encuestas en "L'opinió de les dones recluses a Catalunya sobre l'encarcerament" de la Generalitat de Catalunya. Pág. 7.

${ }^{x v i}$ Porcentajes sobre las personas analizadas, de la totalidad de personas encarceladas en la Comunidad Catalana. En "Les dones a les presons de Catalunya". Pág. 39

xvii SURT, "Violencia contra las mujeres. Análisis en la población penitenciaria femenina". Pág. 11

xviii Todas estas aseveraciones subjetivas forman parte de las manifestaciones recogidas por la autora, como fruto de la dilatada relación profesional de más de 16 años en el campo de la delincuencia femenina. Conocimiento adquirido a través de las entrevistas personales en profundidad desarrolladas desde el ingreso en el establecimiento, de las audiencias personales bajo petición y de numerosas conversaciones informales, reuniones individuales y grupales.

xix Ídem, Pág. 16-20

xx Ídem, Pág. 23. Datos contrastados para el año 2009 por el Instituto de la Mujer "Macroencuesta de violencia contra las mujeres"

${ }^{x x i}$ Descritos y valorados en el trabajo de Claudia Kestermann dentro del "Estudio internacional sobre el régimen penitenciario de mujeres. Evaluación, estudio de necesidades y mejores prácticas." Pág. 30.

xxii Este Establecimiento se creó en 1991 para acoger una población penitenciaria exclusivamente femenina. Posee una capacidad aproximada de 200 plazas para internas penadas en segundo y tercer grado de tratamiento. Dispone también de una Unidad maternal, compuesta por un módulo de madres, Escuela infantil y Unidad Dependiente.

xxiii Concepto utilizado por Toby Herr, director del programa Match, proyecto de apoyo a redes familiares, en Chicago 1985.

${ }^{\text {xxiv }}$ Este aspecto de las prisiones femeninas ha sido muy denostado, por entender que reproducen los roles de género. Sin embargo, estas labores manuales, cuentan con indudables ventajas como tareas de

Revista Española de Investigación Criminológica 
enganche en personas inexpertas y con dificultades motrices ya que además de ser muy gratificantes, no requieren infraestructura compleja ni grandes gastos económicos.

${ }^{x x v}$ Quizás sea la escuela la tarea más gratificante que podamos proponerles, pues es en este espacio cuando muchas mujeres descubren por ellas mismas su capacidad para leer los letreros de las calles, redactar su primera carta, escribir su primera instancia o petición, o leer su correspondencia sin la intermediación de terceros. En niveles superiores acceden a un mundo de conocimiento que abandonaron en la infancia, al haber dejado precozmente la escolarización (por asumir responsabilidades familiares, por su precoz maternidad o por falta de estímulo)

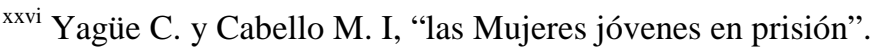

${ }^{\text {xxvii }}$ Se propone para este papel a internas de edad madura de muy bajo perfil delictivo, con estabilidad emocional y responsabilidad. En su papel debe encontrar el equilibrio entre el soporte afectivo, y la instauración de límites precisos a la conducta de su pupila. Controlará la asistencia a actividades y su rendimiento; las relaciones con las demás internas (protegiéndola de robos y abusos o compañías perniciosas); y si fuera preciso, controlará la capacidad de gasto, para evitar un consumo compulsivo irresponsable de su peculio.

${ }^{x x v i i i}$ Los datos que se mencionan provienen de las memorias confeccionadas a final de año, programa o curso escolar por los responsables de ejecución de cada área del centro para su remisión a la Subdirección General de Tratamiento y Gestión y/o Sanidad de la Dirección General de Instituciones Penitenciarias.

${ }^{\text {xxix }}$ Con una participación de 16 internas, 5 de ellas $(31,3 \%)$ completaron el programa, y una sola $(6,3)$ lo abandonó voluntariamente.

${ }^{\mathrm{xxx}}$ Por citar algunos ejemplos. J. es una interna de etnia gitana que, animada por su profesor de educación base, ha escrito un entrañable relato de sus avatares vitales. Debido a su calidad, ha sido solicitado por una editorial para su publicación. M. interna de mediana edad que nunca tuvo un oficio, conocida por sus incontables hurtos al hilo de su drogodependencia, disfruta hoy del primer trabajo remunerado de su vida, en la hostelería; pero su primer éxito personal consistió en descubrir su aptitud hacia la pintura, y exponer sus obras pictóricas en centros culturales de la provincia.

${ }^{x x x i}$ Citamos un caso muy significativo. M. una joven de 21 años incursa en muy diversos sumarios y fuertes condenas, altamente dependiente de la heroína, con alejamiento familiar por robo y agresión a la madre, y con la custodia de su hijo recientemente retirada por los servicios sociales. Tras un periodo inicial de desconcierto, asume el compromiso de participar activamente en los grupos de drogodependencia y de jóvenes. Su principal acicate es recuperar el vínculo con su hijo. Atravesando las diferentes etapas formativas se encuentra desde hace más de un año contratada en un taller productivo del establecimiento con una total entrega y rendimiento. Se ha mediado en las relaciones con la madre, y los servicios sociales, con quienes se está planteando la devolución de la custodia del menor, y su inclusión próxima a un programa de inserción laboral externo.

xxxii En Dünkel F., "Perepectiva- 5 tesis acerca del régimen penitenciario de Mujeres". En "Estudio internacional sobre el régimen penitenciario de mujeres. Evaluación, estudio de necesidades y mejores prácticas”. Universidad Greifswald. 2005 Págs. 43-44 
